\title{
HETEROGENEIDADE COGNITIVA NA DOENÇA DE ALZHEIMER
}

\author{
PAULO ROBERTO DE BRITO-MARQUES*, WVS JOANETTE* *, BERNADETTE SKA**, \\ ARLETE POISSANT ${ }^{* *}$, LAURENCE MELAÇON* ${ }^{*}$, FRANCINE GIROUX**
}

RESUMO - Os autores relatam e discutem aspectos neuro-psicológicos relacionados à organização funcional do cérebro ligados à cognição em 81 pacientes com doença de Alzheimer. Confrontam aspectos clínicos da homogeneidade com os da heterogeneidade, dentro da organizaçāo funcional do cérebro. Foram utilizados para diagnóstico os critérios do NINCDS-ADRDA. Todos os pacientes estavam no início da evoluçăo,nos estágios 3 ou 4 de Reisberg (Functional Assessement Staging-FAST). A conclusāo mostra que existem dois perfis cognitivos: o perfil não recorrente formado da maioria dos pacientes, o qual testemunha a heterogeneidade dependente dos fatores genéticos e ambientais de cada individuo; e o outro, perfil recorrente formado por grupo menor de pacientes, retrata alterações na heterogeneidade de formas clínicas, nosológicas, patológicas e senescentes.

PALAVRAS-CHAVE: Alzheimer, demência.

\section{Cognitive heterogeneity in Alxheimer's disease}

SUMMARY - The authors describe and discuss neuropsichological aspects related to the functional organization of the brain connected to cognition in 81 Alzheimer's disease patients in the Laboratoire Theophile-Alajouanine du Centre de Recherche du Centre hospitalier Côte-des-Neiges in the University of Montreal. They confront the clinical of homogeneity against the heterogeneity within functional organization of the brain. The criteria used for diagnosis were those of the NINCDS-ADRDA. All of the patients were in the begining of the evolution, in stages 3 and 4 of Reiberg (Functional Assessement Staging, FAST). The conclusion shows that there are two cognitive profiles: a non-recurrent profile, made up by the majority of the patients, and the heterogeneity depends on the genetic background of each individual; a recurrent profile, made up by a small group of patients that show changes in the heterogeneity of clinical, nosological, pathological and normal aging forms.

KEY-WORDS: Alzheimer, dementia.

Até início dos anos 80, prevaleceu o conceito de que as manifestações neuropsicologicas encontradas na doença de Alzheimer (DA) eram uniformes e indissociadas. Este conceito era o de uma síndrome afaso-apráxico-agnósica correspondendo a uma lesão da linguagem, dos gestos e das percepções sensoriais. Estas idéias foram popularizadas pelo grupo de Genebra, que se inclinou por esta linha de pensamento por mais de uma década ${ }^{13}$. Os primeiros trabalhos que mostraram a presença de manifestaçōes neuropsicológicas não homogêneas foram de estudos de casos. Assim, certo número de trabalhos tem sido relatado a propósito de pacientes cujo quadro de apresentação clínica não respeita a síndrome afaso-apráxico-agnósica defendida pelo grupo da escola de Genebra ${ }^{3}$. A

*Professor Assistente da Disciplina de Neurologia do Departamento de Medicina Clínica da Faculdade de Ciências Médicas da Universidade de Pernambuco (UPE). Ambulatório de Demências da Seção de Neurologia do Hospital Oswaldo Cruz da UPE. **Laboratoire Théophile-Alajouanine, Centre de Recherche du Centre hospitalier Côte-des-Neiges, Montréal (Québec), Canada. Aceite: 17-janeiro-1995.

Dr. Paulo Roberto de Brito Marques - Rua Santa Terezinha 58, Jardim Atlântico - 53140-170 Olinda PE Brasil. 
importância da identificação precoce da doença tipo Alzheimer (DTA) relaciona-se com medidas terapêuticas e comportamentais. Esta identificação precoce visa retardar a progressão da doença baseada principalmente no seu diagnóstico probabilístico(o diagnóstico final da DA é feito atra- vés de dados clínicos e histopatológicos), a partir de certo número de critérios de inclusão e exclusão ${ }^{10}$.

O objetivo desta apresentação é destacar a questão da heterogeneidade dos perfis cognitivos na DA, em vistas ao caráter quase ilimitado das susceptíveis alterações que poderão vir a lesar esses perfis.

\section{MÉTODO}

O Protocole d'Évaluation Neuropsycologique Optimal (PENO) permitiu apreciar a integridade de vários componentes e subcomponentes cognitivos como: memória (empan de palavras, memória lógica, reprodução visual e evocação facilitada), linguagem (compreensão léxica e semântica, denominação, evocaçảo léxica e capacidade discursiva), gnosias (descriminação visual, emparelhamento semântico, julgamento de orientação de linhas e teste dos sinos), praxias (pantomimas, gestos desprovidos de sentido, desenho figurativo e geométrico e cópia da figura de Rey) e funçōes executivas(teste de Stroop e torre de Londres).

As performances das pessoas foram submetidas a análises hierárquicas em cadeia, a fim de identificar os subgrupos de pacientes com DTA caracterizados por diferentes perfis cognitivos. Diversas medidas de distância (euclidiana, cosseno) e métodos de semelhanças (simples, Ward) foram utilisados para confirmar os melhores reagrupamentos destes pacientes.

\section{MATERIAL}

Oitenta e um pacientes com DTA (critérios do NINCDS-ADRDA) ${ }^{10}$ no início da evolução(estágios 3 ou 4 do Functional Assessement Staging-FAST de Reisberg) ${ }^{12}$ avaliados pelo PENO do Laboratoire ThéophileAlajouanine, Montréal (Québec) Canada.

\section{RESULTADOS}

Os resultados mostraram a divisão dos pacientes em 14 sub- grupos: 3 subgrupos comportando grande número de pacientes ( $15<n<22), 4$ subgrupos com número médio de pacientes $(2<n<5)$ e 7 subgrupos compostos cada um de um único paciente $(n=1)$, (Tabela). Cada um destes últimos subgrupos se caracterizou especificamente em função da lesão do perfil cognitivo que lhe é próprio. O número das lesōes dos perfis cognitivos apareceu como se fosse ilimitado na DTA. Todavia, este

Tabela. Heterogeneidade cognitiva dos subgrupos dos 81 pacientes com DTA nos estágios 3 ou 4 de Reisberg.

\begin{tabular}{cc}
\hline Grande subgrupo & Número de pacientes \\
1 & 22 \\
2 & 22 \\
3 & 15
\end{tabular}

Médio subgrupo

$\begin{array}{ll}1 & 2 \\ 2 & 5 \\ 3 & 5 \\ 4 & 3\end{array}$

Pequeno subgrupo

\begin{tabular}{ll}
1 & 1 \\
1 & 1 \\
1 & 1 \\
1 & 1 \\
1 & 1 \\
1 & 1 \\
\hline
\end{tabular}
grande número de perfis diferentes pode ser explicado pela presença de dois tipos de perfis cognitivos: a existência de um número restrito de perfis recorrentes e a presença de um número maior de perfis não recorrentes.

\section{COMENTÁRIOS}

Se por um lado a experiência clínica mostrou que todos os pacientes com DTA chegam a fase terminal com o mesmo quadro cognitivo, por outro lado estes mesmos pacientes quando começam precocemente a DTA apresentam quadro clínico heterogeneamente variado, contendo manifestações clinicas diversas ${ }^{14}$. Entretanto, $o$ atual debate acerca da heterogeneidade repousa sobre os trabalhos efetuados por Martin e col. ${ }^{9}$ e Neary e col. ${ }^{11}$ sobre grupos de pacientes com diversos aspectos de seu funcionamento cognitivo. Uma 
outra forma de poder abordar o problema da heterogeneidade das manifestações neuropsicológicas da DTA é estudar uma série de pacientes confrontando seus resultados. Num chamado estudo de casos múltiplos realizado por Caramazza², ele teve cuidado para não haver interferência na variabilidade entre pacientes, no momento da análise das tendências centrais de um subgrupo de pacientes.

Nos quadros demenciais,como na heterogeneidade encontrada na DTA, várias manifestações clínicas diferentes entre si encontram-se em subgrupos funcionais que são identificados a partir de exame completo das habilidades cognitivas como : memória, linguagem, gnosia, praxia e funções executivas ${ }^{1.8}$. Em outro trabalho recente, Ska e col. ${ }^{15}$ lembram que a heterogeneidade cognitiva chega a existir dentro de uma mesma função, a qual é chamada de subcomponente. O componente linguagem, por exemplo, não pode ser afetado de modo homogêneo, pois a maioria dos pacientes com DTA apresenta perfil de lesāo léxico-semântico> sintático>fonológico; uma minoria destes pacientes apresenta-se de modo inverso. Estes dados se consolidam na subdivisão dos perfis cognitivos. Os perfis não recorrentes relacionam-se com influências interacionais existentes entre diversos fatores que estão ligados à natureza precisa da organização funcional cerebral de um determinado indivíduo. Esta organizaçāo funcional do cérebro depende da bagagem genética (sexo, dominância manual) e da exposição ambiental (grau de penetração da linguagem escrita). Lesões focais na organização do funcionamento cognitivo de um indivíduo podem ser expressas por diferentes perfis de lesão cognitiva. Isto é, numa mesma lesão cercbral não aparecerão necessariamente as mesmas lesōes cognitivas, por exemplo, em função da idade, sexo e escolaridade. Os perfis não recorrentes poderiam traduzir os efeitos de uma deterioração cerebral em forte interação com os próprios fatores peculiares a cada pessoa. Deste modo, também não poderemos excluir a possibilidade de que alguns destes indivíduos, portadores dos testemunhos de seu perfil cognitivo, venham desenvolver a DTA. Nos perfis recorrentes, além das diferenças interindividuais, eles poderiam expressar diferentes formas clínicas da DTA. Estes mesmos perfis clínicos poderiam também expressar diferentes formas nosológicas, patológicas e senescentes na DTA. O número de perfís clínicos da DA é quase ilimitado em razão da interação entre as fontes de heterogeneidade da doença por um lado e, do outro, do conjunto dos fatores susceptíveis de ser a fonte de diferenças interindividuais do que diz respeito à funçōes cognitivas?.

Uma das implicações da presença de uma heterogeneidade dos perfis cognitivos na DTA é a dificuldade que esta condição possui para identificar os procedimentos que visam o diagnóstico precoce da DA. Existem na DTA habilidades cognitivas que são afetadas de modo privilegiado servindo como marcadores cognitivos. Recentemente Brito-Marques e col.' apresentaram uma avaliação completa da heterogeneidade inter e intra-funcional da organização cerebral, verificando seus subcomponentes, a qual poderá demonstrar tais marcadores cognitivos presentes na fase inicial da DTA. Entre outros, os procedimentos de avaliação breve, como o Mini-Mental State Examination ${ }^{4}$, não permitem detectar todos os pacientes com DTA no início de sua evolução, apesar do interesse que este teste continua despertando na atenção dos clínicos.

Em conclusão, é necessário chamar a atenção para o que segue. A presente síntese procura salientar a importância atribuída ao estudo dos aspectos neuropsicológicos da DA e a melhor compreensão da heterogeneidade das manifestações cognitivas de subgrupos de doentes caracterizados por um determinado perfil cognitivo em um dado momento de seu comprometimento.

As repercussões clínicas e teóricas desses argumentos já foram constatadas o suficiente para garantir esta visão cognitiva encontrada na organizaçāo funcional do cérebro de pacientes com DA.

Para finalizar, é de bom alvitre condensar algumas aspectos essenciais dos perfis recorrentes que foram encontrados ao longo deste estudo:

1. Heterogeneidade da organização funcional do cérebro (lesões iguais e topograficamente semelhantes não apresentam necessariamente as mesmas manifestações cognitivas). 
2. Heterogeneidade das alterações neuropatológicas (o grau do hipometabolismo cerebral é encontrado de modo variável num mesmo hemisfério, não traduzindo uma correlação entre as heterogeneidades neuropatológicas e neuropsicológicas).

3. Existência de formas distintas da doença como: de início precoce/tardio, de evolução lenta/ rápida, de caráter familiar/esporádica.

4. Exarcebação de uma heterogeneidade presente no senescente - pode ocorrer em virtude de já existir no idoso normal uma heterogeneidade da organização funcional do cérebro ligada à cogniçāo (como existe na população adulta). A idade como fator de risco, virtualmente poderá alterar a organização funcional deste cérebro dando início ao processo mórbido.

\section{REFERÊNCIAS}

1. Brito-Marques PR, Joanette Y, Poissant A, Ska B. Avaliaçāo neuropsicológica adequada às demências. Arq Neuropsiquiatr 1995, 1:147-152.

2. Caramazza A. On drawing inferences about the structure of cognitive systems from the analysis of patterns of impaired performance: the case for single-patient studies. Brain and Cognition 1986, 5:565-582.

3. Crystal HA, Horoupian DS, Katzman R, Jotkowitz S. Biopsy-proved Alzheimer disease presenting as right parietal lobe syndrome. Ann Neurol 1981, 12:186-188.

4. Folstein MF, Folstein SE, McHugh PR. Mini Mental State: a pratical method for grading the cognitive state of patients for the clinician. J Psychiatr Res 1975, 12:189-198.

5. Joanette Y, Ska B, Poissant A, Béland R. Neuropsychological aspects of Alzheimer's disease: evidence for inter and intra -function heterogeneity. In Boller F, Forette F. Khachaturian Z, Poncet M, Christien Y(eds). Heterogeneity of Alzheimer's disease New York: Springer-Verlag . 1992, p 33-42.

6. Joanette Y, Ska B, Poissant A, Giroux F. Multiple neuropsy- chological profiles among early demented patients of the Alzheimer type. TENNET IV 1993, Montreal, 12-14 mai.

7. Joanette Y, Ska B, Poissant A, Melançon L. Manifestations neuropsychologiques dans les démences de type Alzheimer: de l'homogénéité à l'hétérogénéité. Alzheimer Actualités 1992, 92:4-7.

8. Joanette $Y$, Poissant A, Valdois S. Neuropsychological dissociations in dementia of the Alzheimer type: a multiple single case study. J Clin Exp Neuropsychol 1989, 11:91.

9. Martin A, Brouwers P, Lalonde F, Cox C, Teleska P, Fedio P, Foster NL, Chase TN. Toward a behavioral typology of Alzheimer's patients. J Clin Exp Neuropsychol 1986, 8:594-610.

10. McKhann G, Drachman D, Folstein M, Katzman R, Price D, Stadlan EM. Clinical diagnosis of Alzheimer's disease: report of the NINCDS-ADRDA Work Group under the auspices of Departament of Health and Human Services Task Force on Alzheimer's disease. Neurology 1984, 34:939-944.

11. Neary D, Snowden JS, Bowen DM, Sims NR, Mann D, Benton JS, Northen B, Yates P, Avision D. Neuropsychogical syndromes in presenile dementia due to cerebral atrophy. J Neurol Neurosurg Psychiatry 1986, 49:163-174.

12. Reisberg B. Ferris SH, DeLeon MJ, Crook T. The global deterioration scale for assessement of primary degenerative dementia. Am J Psychiatry 1982, 139:1136-1139.

13. Richard J, Constantinidis J. Les démences de la vieillesse: notions acquises et problèmes cliniques actuels. Confront Psychiatrique 1970, 3:39-61.

14. Schwartz MF. Introduction to chapter 5: Neuropsychology of Alzheimer's disease: the case for subgrups by A. Martib. In Schwartz MJ (ed): Modular deficits in Alzheimer-type Dementia. Cambridge (Mass): MIT Press, 1990, p 143.

15. Ska B, Joanette $Y$, Poissant A, Béland R, Lecours AR. Language disorders in dementia of the Alzheimer type: contrastive patterns from a multiple single case study (Abstr). Academy of Aphasia 28th Annual Meeting. Baltimore (USA), 1990, October 21-23. 
CORRIGENDA. O Editor recebeu carta do Professor Yves Joanette fazendo notar que, em respeito a cada um dos colaboradores e por uma questão de equidade, a ordem dos autores seja mudada em dois artigos de que é o pesquisador responsável; faz notar também que os artigos parecem ter redação difícil de compreender e contêm expressões não habitualmente utilizadas em neurologia do comportamento. Esses artigos são os seguintes: Brito-Marques PR, Joanette Y, Poissant A, Ska $B$. Avaliaçâo neuropsicológica adequada às demências [Optimal neuropsychological evaluation of dementias]. Arq Neuropsiquiatr 1995;53(1):147-152; Brito-Marques PR, Joanette Y, Ska B, Poissant A, Melaçon L, Giroux F. Heterogeneidade cognitiva na doença de Alzheimer [Cognitive heterogeneity in Alzheimer's disease]. Arq Neuropsiquiatr 1995;53(3-A):451-454.

Em relação ao primeiro aspecto e dentro das normas de publicações científicas (ver International Committee of Medical Joumal Editors. Uniform requirements for manuscripts submitted to biomedical journals. $N$ Engl J Med 1991;324:424-428) o Editor considera justo o ponto de vista do Professor Yves Joanette e adota a ordem de autores por ele sugerida como chefe-de-escola. $O$ Editor deu ciência do fato ao Dr. Paulo Roberto de Brito Marques que aceitou a resolução. Assim, esses artigos passam a ser catalogados como segue:

- Joanette Y, Poissant A, Ska B, Brito-Marques PR. Avaliação neuropsicológica adequada às demências [Optimal neuropsychological evaluation of dementias]. Arq Neuropsiquiatr 1995;53(1):147-152;

- Joanette Y, Ska B, Poissant B, Melançon L, Giroux F, Brito-Marques PR. Heterogeneidade cognitiva na doença de Alzheimer [Cognitive heterogeneity in Alzheimer's disease]. Arq Neuropsiquiatr 1995;53(3-A):451-454.

Em relação ao segundo aspecto e de acordo com as mesmas normas lembradas no parágrafo anterior, o Editor convidou o Professor Yves Joanette a escrever um editorial em que sejam salientadas as contribuições dele e de sua escola a propósito do tema, com o qual as dificuldades e imprecisões serão naturalmente dirimidas. O Editor espera do Professor Yves Joanette a gentileza de aceitar o convite. Esse editorial seguramente representará importante contribuição ao periódico e aos seus leitores, em particular aos especialistas em neurologia do comportamento. $O$ Editor deixa registrado que, o editoral será publicado assim que recebido. ANTONIO SPINA-FRANÇA, Editor. 